\title{
Quark seesaw, vectorlike fermions and diphoton excess
}

\section{P.S. Bhupal Dev, ${ }^{a}$ Rabindra N. Mohapatra ${ }^{b}$ and Yongchao Zhang ${ }^{c}$}

${ }^{a}$ Max-Planck-Institut für Kernphysik, Saupfercheckweg 1, D-69117 Heidelberg, Germany

${ }^{b}$ Maryland Center for Fundamental Physics, Department of Physics, University of Maryland, College Park, MD 20742, U.S.A.

${ }^{c}$ Service de Physique Théorique, Université Libre de Bruxelles, Boulevard du Triomphe, CP225, 1050 Brussels, Belgium

E-mail: bhupal.dev@mpi-hd.mpg.de, rmohapat@umd.edu, yongchao .zhang@ulb.ac.be

ABSTRACT: We present a possible interpretation of the recent diphoton excess reported by the early $\sqrt{s}=13 \mathrm{TeV}$ LHC data in quark seesaw left-right models with vectorlike fermions proposed to solve the strong $C P$ problem without the axion. The gauge singlet real scalar field responsible for the mass of the vectorlike fermions has the right production cross section and diphoton branching ratio to be identifiable with the reported excess at around $750 \mathrm{GeV}$ diphoton invariant mass. Various ways to test this hypothesis as more data accumulates at the LHC are proposed.

Keywords: Beyond Standard Model, Quark Masses and SM Parameters

ARXIV EPRINT: 1512.08507 


\section{Contents}

1 Introduction 1

2 Scalar sector 3

3 Production and decay of the singlet 3

4 High-scale validity $\quad 9$

$\begin{array}{lll}5 & \text { Discussions } & 10\end{array}$

6 Conclusion 11

A Exact formulae for the $\gamma Z$ and $Z Z$ channel 12

\section{Introduction}

In the early run II data from the $\sqrt{s}=13 \mathrm{TeV}$ Large Hadron Collider (LHC), both CMS [1] and ATLAS [2] experiments have reported an excess of $\gamma \gamma$ events over the SM background with invariant mass around $750 \mathrm{GeV}$. The signal cross section is reported to be $(6 \pm 3) \mathrm{fb}$ by CMS [1] and $(10 \pm 3)$ fb by ATLAS [2]. While this excess has a local statistical significance of around $2.6 \sigma$ (CMS) to $3.9 \sigma$ (ATLAS) and needs more data to firmly rule out the possibility of a statistical fluctuation, it has nonetheless generated a great deal of recent activity in the theory community as a possible signal of beyond the Standard Model (SM) physics and many possible interpretations have been advanced; for a non-exhaustive list of ideas and speculations, see [3-98]. In this note we add another one in the context of a theory proposed many years ago as a solution to the strong $C P$ problem without an axion $[99,100]$.

The model is based on the assumption that there exist $\mathrm{TeV}$-scale vectorlike fermions which are responsible for the seesaw masses for the quarks and charged leptons [101-104] in the context of a left-right (LR) symmetric model based on the gauge group $\mathrm{SU}(3)_{C} \times$ $\mathrm{SU}(2)_{L} \times \mathrm{SU}(2)_{R} \times \mathrm{U}(1)_{B-L}[105-107]$. The SM fermions and the vectorlike fermions are assigned to the gauge group as follows:

$$
\begin{gathered}
\text { SM fermions : } Q_{L, R}=\left(\begin{array}{l}
u \\
d
\end{array}\right)_{L, R}, \quad \psi_{L, R}=\left(\begin{array}{c}
\nu \\
e
\end{array}\right)_{L, R} ; \\
\text { Vectorlike fermions: } P\left(\mathbf{3}, \mathbf{1}, \mathbf{1},+\frac{4}{3}\right), \quad N\left(\mathbf{3}, \mathbf{1}, \mathbf{1},-\frac{2}{3}\right), \quad E(\mathbf{1}, \mathbf{1}, \mathbf{1},-2) .
\end{gathered}
$$

The Higgs sector of the model consists of $\mathrm{SU}(2)_{L, R}$ doublets $\chi_{L, R}$ which break the left and right $\mathrm{SU}(2)$ 's and a real singlet $S$ that gives mass to the vectorlike fermions. An 
appropriate discrete $Z_{2}$ symmetry forbids the bare mass of the vectorlike fermions. Under this $Z_{2}$ symmetry, the Higgs fields $\chi_{L}$ and $S$ are odd as are the right-handed (RH) chirality of the vectorlike fermions; all other fields are even. The Yukawa couplings are given in this case by the Lagrangian

$$
\begin{aligned}
-\mathcal{L}_{Y}= & y_{U} \bar{Q}_{L} \tilde{\chi}_{L} P_{R}+y_{D} \bar{Q}_{L} \chi_{L} N_{R}+y_{E} \bar{L} \chi_{L} E_{R}+(L \leftrightarrow R) \\
& +f_{U} \bar{P}_{L} S P_{R}+f_{D} \bar{N}_{L} S N_{R}+f_{E} \bar{E}_{L} S E_{R}+\text { H.c. }
\end{aligned}
$$

where $\tilde{\chi}_{L, R}=i \sigma_{2} \chi_{L, R}^{*}\left(\sigma_{2}\right.$ being the second Pauli matrix), and $y_{F}, f_{F}$ (with $F=U, D, E$ ) are the Yukawa couplings with potential beyond SM $C P$ violations. Once both the doublets and the singlet obtain their non-vanishing vacuum expectation values (VEVs) $v_{L}, v_{R}, v_{S}$ respectively, we get the seesaw form for the $2 \times 2$ mass matrix for a single quark or lepton flavor:

$$
\mathcal{M}_{F}=\left(\begin{array}{cc}
0 & \frac{1}{\sqrt{2}} y_{F} v_{L} \\
\frac{1}{\sqrt{2}} y_{F} v_{R} & f_{F} v_{S}
\end{array}\right),
$$

which generates the small fermion masses in the SM (except the top-quark mass) and alleviates the hierarchy problem in the Yukawa couplings:

$$
m_{F} \simeq \frac{y_{F}^{2} v_{L} v_{R}}{2 f_{F} v_{S}}
$$

Both the two new VEVs are assumed to be at the (multi-) TeV scale, whereas $v_{L} \simeq$ $246.2 \mathrm{GeV}$ is the electroweak scale. Clearly, the simple seesaw mass formula in eq. (1.4) is not a good approximation for the top quark, as it is expected that the matrix entries $y_{F} v_{R}$ and $f_{F} v_{S}$ are of similar magnitude, which implies a large "right-handed" mixing of the top quark and its partner through $\sin \alpha_{R}^{t} \sim \frac{1}{\sqrt{2}} y_{T} v_{R} / f_{T} v_{S}$. Therefore in general, one should take into consideration the whole $2 \times 2$ mass matrix (1.3) and diagonalize $\mathcal{M}_{F} \mathcal{M}_{F}^{\dagger}$ to get the mass eigenvalues of the SM quarks and their partners.

As far as the flavor structure and quark mixing are concerned, we can have either heavy quarks with degenerate masses (respectively for the up and down type flavors) in which case the SM quark mixings are completely determined by the flavor structure of the Yukawa couplings $y_{U, D}$ [108], or the couplings $y_{U, D}$ are hierarchical but diagonal (e.g. from some discrete symmetry assignments) and the matrices $f_{U, D}$ are of order $\mathcal{O}(1)$ in which case we have flavor anarchic [109].

As a direct result of the Lagrangian in (1.2), the heavy vectorlike quarks decay dominantly to the SM gauge and Higgs bosons plus SM quarks, especially for the top and bottom partners. Due to the Goldstone equivalence theorem, the branching ratios for the decays to $W, Z$ and Higgs are approximately $2: 1: 1$. The current LHC constraints put a $95 \%$ confidence level (CL) lower limit on the top partner mass from $715-950 \mathrm{GeV}$ and on the bottom partner mass from $575-813 \mathrm{GeV}$ [110], depending on their decay branching ratios. These lower bounds are much stronger than those from the flavor changing neutral currents (FCNCs) mediated by the heavy quarks [108]. 


\section{Scalar sector}

The gauge and $Z_{2}$-invariant scalar potential including the singlet is given by

$$
\begin{aligned}
V= & -\mu_{L}^{2} \chi_{L}^{\dagger} \chi_{L}-\mu_{R}^{2} \chi_{R}^{\dagger} \chi_{R}-\frac{1}{2} \mu_{S}^{2} S^{2}+\lambda_{1}\left[\left(\chi_{L}^{\dagger} \chi_{L}\right)^{2}+\left(\chi_{R}^{\dagger} \chi_{R}\right)^{2}\right]+\lambda_{2}\left(\chi_{L}^{\dagger} \chi_{L}\right)\left(\chi_{R}^{\dagger} \chi_{R}\right) \\
& +\lambda_{S} S^{4}+\lambda_{3} S^{2}\left(\chi_{L}^{\dagger} \chi_{L}+\chi_{R}^{\dagger} \chi_{R}\right) .
\end{aligned}
$$

As in [108] we keep in the potential the mass terms $\mu_{L, R}^{2}$ different so that they break the LR symmetry softly. Among all the terms given above, the $\lambda_{2}$ term mixes the left and right-handed doublets and the $\lambda_{3}$ term couples the singlet $S$ to the doublets. The mass square matrix for the scalars after minimization of the potential is given by

$$
\mathcal{M}_{\text {scalar }}^{2}=\left(\begin{array}{ccc}
-\mu_{L}^{2}+3 \lambda_{1} v_{L}^{2} & \lambda_{2} v_{L} v_{R} & 2 \lambda_{3} v_{L} v_{S} \\
\lambda_{2} v_{L} v_{R} & -\mu_{R}^{2}+3 \lambda_{1} v_{R}^{2} & 2 \lambda_{3} v_{R} v_{S} \\
2 \lambda_{3} v_{L} v_{S} & 2 \lambda_{3} v_{R} v_{S} & -\mu_{S}^{2}+6 \lambda_{S} v_{S}^{2}
\end{array}\right) .
$$

The FCNC constraint on the $W_{R}$ boson from $K$-meson mixing implies a lower bound on the VEV $v_{R}$ (which depends also on the gauge coupling $g_{R}$ ) [111], and is much larger than the SM VEV $v_{L}$. Then the mixing between the SM Higgs $h$ and its heavy RH partner $H$ is suppressed by the ratio $\lambda_{2} v_{L} / 2 \lambda_{1} v_{R}$ [108]. The $h-S$ mixing is expected to be of order $\lambda_{3} v_{L} / \lambda_{S} v_{S}$. On the contrary, as long as $\lambda_{3} \sim \lambda_{S}$, under the condition of $v_{S} \sim v_{R}$ at the (multi-) TeV scale, $H$ and $S$ can potentially have sizable mixing. Then in this case the singlet $S$ can decay into the SM top quark, $W$ and $Z$ gauge bosons and SM Higgs pairs by the large mixing to $H$ [108]. It is also subject to some constraints from flavor observations $[112,113]$, precision tests $[114,115]$ and neutrinoless double beta decay [116]. To explain the $750 \mathrm{GeV}$ diphoton excess, we make the simple choice that the mixing term $\lambda_{3}$ at tree level is very small, so that the SM Higgs observables [117-119] and the electroweak vacuum stability analysis [108] do not get affected much by the presence of $S$.

\section{Production and decay of the singlet}

The dominate production channel for the singlet $S$ at the LHC is the gluon-gluon fusion process mediated by the $\mathrm{TeV}$-scale vectorlike fermions in the triangle loop. As the heavy fermion couplings to $S$ are proportional to their masses, it is expected that all three generations of heavy fermions contribute significantly to the gluon fusion loops, which enhances the production cross section by roughly a factor of $N_{f}^{2}$ (with $N_{f}$ being the number of flavors). At $\sqrt{s}=13 \mathrm{TeV}$, the cross section for gluon fusion channel for a $750 \mathrm{GeV}$ scalar with SM Higgs-like couplings is $\sigma_{0}^{13 \mathrm{TeV}} \simeq 850 \mathrm{fb}$, taking into account the large $k$-factor of order 2 [120]. The corresponding production cross section of the singlet $S$ in our model can be obtained easily by rescaling the loop factor $A_{1 / 2}$ and the Yukawa couplings and masses in the fermion loop: $\sigma(g g \rightarrow S)^{13 \mathrm{TeV}}=\sigma_{0}^{13 \mathrm{TeV}} r$, where the scaling factor is defined as

$$
r=\left|\frac{f_{T} \sin \alpha_{L}^{t} \sin \alpha_{R}^{t}}{M_{t} / v_{L}}+\sum_{F=U, D} \frac{f_{F} v_{L}}{M_{F}} \frac{A_{1 / 2}\left(\tau_{F}\right)}{A_{1 / 2}\left(\tau_{t}\right)}\right|^{2},
$$


with the first term on the r.h.s. for the top quark loop (with $M_{t} \simeq 173.2 \mathrm{GeV}$ ) and the second term for all the heavy vectorlike quark partners. Here $\tau_{F}=M_{S}^{2} / 4 M_{F}^{2}$ and the fermion loop function is given by (see e.g. [121])

$$
A_{1 / 2}(\tau)=2[\tau+(\tau-1) f(\tau)] \tau^{-2},
$$

where the function $f(\tau)$ is defined as

$$
f(\tau)=\left\{\begin{array}{cc}
\arcsin ^{2} \sqrt{\tau} & (\text { for } \tau \leq 1) \\
-\frac{1}{4}\left[\log \left(\frac{1+\sqrt{1-1 / \tau}}{1-\sqrt{1-1 / \tau}}\right)-i \pi\right]^{2} & (\text { for } \tau>1) .
\end{array}\right.
$$

In the parameter space of interest, the top quark contribution is suppressed by the LH mixing

$$
\sin \alpha_{L}^{t} \simeq \frac{y_{T} v_{L}}{\sqrt{2} f_{T} v_{S}} \sim 0.1
$$

where we have chosen $v_{S} \sim 1.2 \mathrm{TeV}$, as is required to explain the diphoton excess (see figure 4 below) and the Yukawa parameter $y_{T}$ is given by

$$
y_{T}=\sqrt{\frac{2 M_{t} M_{T}}{v_{L} v_{R}}} \sim \mathcal{O}(1) .
$$

On the other hand, due to the large Yukawa coupling and $v_{R}$, the $\mathrm{RH} t-T$ mixing is generally very large, of order one, and the top partner mass is largely from or even dominated by the $v_{R}$ term

$$
M_{T}^{2}=\frac{1}{2} y_{T}^{2}\left(v_{R}^{2}+v_{L}^{2}\right)+f_{T}^{2} v_{S}^{2}-M_{t}^{2} .
$$

For a typical value of $v_{R}=3 \mathrm{TeV}$ and $v_{S}=1 \mathrm{TeV}$, the top partner mass can reach up to $2.5 \mathrm{TeV}$. The $\mathrm{LH}$ and $\mathrm{RH}$ mixing of other SM quarks and their corresponding heavy partners are comparatively much smaller and can be safely neglected, for which $M_{F} \simeq f_{F} v_{S}$ with $f_{F}$ a universal Yukawa coupling for all the flavors of vectorlike quarks and leptons in the Lagrangian (1.2). ${ }^{1}$ The production of $S$ is then predominately from these five heavy quark loops in eq. (3.1). For a fixed value of $f_{F} \sim \mathcal{O}(1)$, the production cross section is suppressed by the heavy fermion masses via approximately $\sigma(g g \rightarrow S) \propto M_{F}^{-2}$.

As far as the decay of $S$ is concerned, due to our choice of parameters, it couples dominantly to the heavy vectorlike fermions with a coupling $f_{F}$ of order one. All the couplings of $S$ to other particles are from mixing or radiative effects. The largest coupling to the SM fermions is to the top quark from $t-T$ mixing, i.e. $f_{T} \sin \alpha_{L}^{t} \sin \alpha_{R}^{t}$, which is however suppressed by the small LH mixing $\sin \alpha_{L}^{t}$. For loop-induced channels, $S$ can decays into $g g, \gamma \gamma, \gamma Z$ and $Z Z$ mainly via the large number of heavy quark and lepton loops. As the SM $W$ boson does not couple directly to the heavy fermions, the $S \rightarrow W W$

\footnotetext{
${ }^{1}$ For the bottom quark partner, the difference $M_{B}-f_{B} v_{S}$ is only $26 \mathrm{GeV}$ for $v_{R}=3 \mathrm{TeV}$ and $f_{B} v_{S}=$ $1 \mathrm{TeV}$, for instance. For other quark and lepton flavors the mixing effects are even smaller.
} 
channel is dominantly induced from the top quark loop which is comparatively suppressed by the small mixing $\sin \alpha_{L}^{t}$. On the other hand, the $Z$ boson couples to the heavy vectorlike fermions with couplings proportional to $Q_{F} s_{w}^{2}$, where $Q_{F}$ is the electric charge of heavy fermions and $s_{w} \equiv \sin \theta_{w}, \theta_{w}$ being the SM weak mixing angle. As $M_{S} \gg 2 M_{Z}$, it is expected that the $\gamma \gamma, \gamma Z$ and $Z Z$ decay channels have comparable fractional widths

$$
\Gamma_{\gamma \gamma}: \Gamma_{Z \gamma}: \Gamma_{Z Z}=1: 2 \tan ^{2} \theta_{w}: \tan ^{4} \theta_{w}
$$

which is a clean signal of vectorlike fermions. Combining all these decay channels, we find that since they are suppressed either by the small mixing or by the loop factors, the width of hidden singlet $S$ is rather small, as we will show below.

The various partial decay widths of $S$ from tree and loop-level interactions are given by

$$
\begin{aligned}
& \Gamma_{t \bar{t}}=\frac{3 y_{S t \bar{t}}^{2} M_{S}}{16 \pi}\left(1-\frac{4 M_{t}^{2}}{M_{S}^{2}}\right)^{3 / 2}, \\
& \Gamma_{g g}=\frac{\alpha_{s}^{2} M_{S}^{3}}{128 \pi^{3}}\left|\sum_{F=t, U, D} \frac{f_{F}}{M_{F}} A_{1 / 2}\left(\tau_{F}\right)\right|^{2}\left(1+k_{g g} \frac{\alpha_{s}}{\pi}\right), \\
& \Gamma_{\gamma \gamma}=\frac{\alpha^{2} M_{S}^{3}}{256 \pi^{3}}\left|\sum_{F=t, U, D, E} \frac{f_{F}}{M_{F}} N_{C_{F}} Q_{F}^{2} A_{1 / 2}\left(\tau_{F}\right)\right|^{2}, \\
& \Gamma_{\gamma Z} \simeq \frac{\alpha^{2} M_{S}^{3}}{128 \pi^{3} s_{w}^{2} c_{w}^{2}}\left|\sum_{F=t, U, D, E} \frac{f_{F}}{M_{F}} N_{C_{F}} Q_{F}\left(\frac{1}{2} I_{3_{F}}-Q_{F} s_{w}^{2}\right) A_{1 / 2}\left(\tau_{F}\right)\right|^{2}, \\
& \Gamma_{Z Z} \simeq \frac{\alpha^{2} M_{S}^{3}}{256 \pi^{3} s_{w}^{4} c_{w}^{4}}\left|\sum_{F=t, U, D, E} \frac{f_{F}}{M_{F}} N_{C_{F}}\left(\frac{1}{2} I_{3_{F}}-Q_{F} s_{w}^{2}\right)^{2} A_{1 / 2}\left(\tau_{F}\right)\right|^{2} .
\end{aligned}
$$

For the $\gamma Z$ and $Z Z$ channels we show the formulae in the massless $Z$ boson limit in which we can see clearly the relation given in eq. (3.7). The exact expressions for these two channels are given in the appendix. In eqs. (3.8)-(3.12), $\tau_{F}=M_{S}^{2} / 4 M_{F}^{2}, y_{S t \bar{t}}=\sqrt{2} f_{T} \sin \alpha_{L}^{t} \sin \alpha_{R}^{t}$ and $k_{g g}=\frac{95}{4}-\frac{7 N_{f}}{6}$ (with $N_{f}=6$ ) is the next-to-leading order factor for the gluon decay channel. The strong coupling $\alpha_{s} \equiv g_{s}^{2} / 4 \pi$ and the fine-structure constant $\alpha \equiv e^{2} / 4 \pi$ are evaluated at the resonance scale of $750 \mathrm{GeV}$. The loops run over the top and heavy vectorlike fermions for which the effective Yukawa couplings $f_{F}$ are respectively $f_{T} \sin \alpha_{L}^{t} \sin \alpha_{R}^{t}$ (top quark), $f_{T} \cos \alpha_{R}^{t}$ (top partner) and $f_{F}$ (all other heavy fermions).

The total decay width of the scalar $S$ is then given by

$$
\Gamma_{S} \simeq \Gamma_{t \bar{t}}+\Gamma_{g g}+\Gamma_{\gamma \gamma}+\Gamma_{\gamma Z}+\Gamma_{Z Z}
$$

In the minimal LR seesaw model, only the universal Yukawa coupling $f_{F}$ and the VEV $v_{S}$ are input by hand, apart from the RH scale $v_{R}$. The total decay width for some typical values of $v_{R}$ is shown in figure 1 as a function of the VEV $v_{S}$. For this and subsequent plots, we have set the universal Yukawa coupling $f_{F}=1$, unless otherwise specified. Note that the total width of $S$ is at most a few $\mathrm{GeV}$ and decreases with higher $v_{S}$. Therefore, if this model is right, $S$ will always appear as a narrow resonance at the LHC. This prediction 


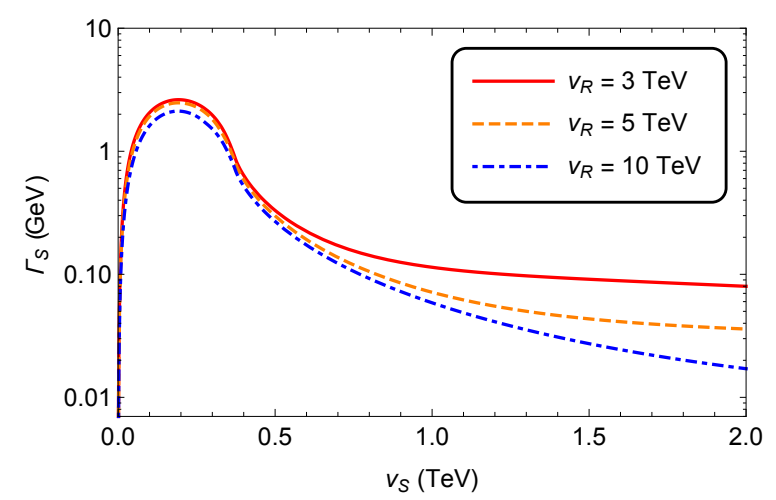

Figure 1. Total width of the scalar $S$ in the LR seesaw model, as a function of the singlet VEV $v_{S}$. The different curves are for $v_{R}=3,5$ and $10 \mathrm{TeV}$. Here we have set $f_{F}=1$.
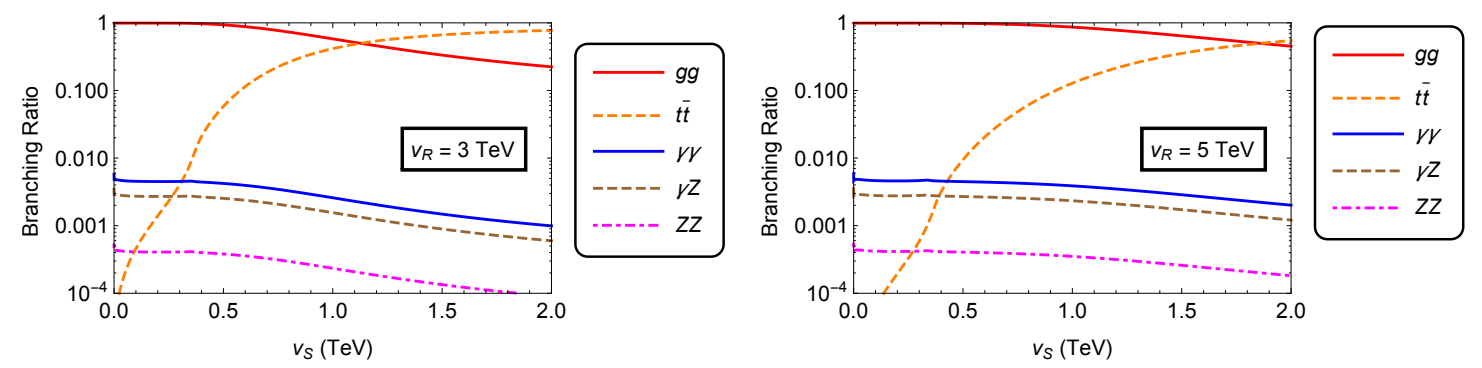

Figure 2. Branching ratios of the scalar $S$ into $t \bar{t}, g g, \gamma \gamma, \gamma Z$ and $Z Z$ final states in the LR seesaw model, as functions of the singlet $\mathrm{VEV} v_{S}$. The two panels are for $v_{R}=3$ and $5 \mathrm{TeV}$ respectively.

can be easily tested in near future. In particular, if the wide resonance behavior of the reported excess with $\Gamma_{S} \sim 45 \mathrm{GeV}$ as claimed by ATLAS [2] persists with more data, the minimal version of this model will be ruled out.

The various branching ratios for the channels discussed above are shown in figure 2 as a function of the VEV $v_{S}$ for $v_{R}=3$ and $5 \mathrm{TeV}$. Comparing the different decay channels, we find that in most of the parameter space of interest, $g g$ is the dominate decay channel. The $t \bar{t}$ channel depends quadratically on the Yukawa coupling and increases when $v_{S}$ is larger, which can be easily seen from the seesaw relation (1.4). For $v_{S} \sim 1 \mathrm{TeV}$ it is even comparable with the loop-induced $g g$ channel. The decay rates into SM gauge bosons $\gamma \gamma$, $\gamma Z$ and $Z Z$ are suppressed by both the gauge coupling $\alpha^{2} / \alpha_{s}^{2}$ and loop factors and are at the per mil level or even smaller.

The diphoton signal cross section $\sigma_{\gamma \gamma} \equiv \sigma(g g \rightarrow S) \times \mathrm{BR}(S \rightarrow \gamma \gamma)$ as function of $v_{S}$ is shown in figure 3 for different values of the $\mathrm{RH}$ scale $v_{R}=3,5$ and $10 \mathrm{TeV}$. The horizontal (green) shaded region shows the preferred range of the observed diphoton signal: $\sigma_{\gamma \gamma}^{\text {obs }}=(8 \pm 5) \mathrm{fb}[1,2]$. The vertical (orange) shaded region is the 95\% CL exclusion region from direct searches for the bottom partner at the $\sqrt{s}=8 \mathrm{TeV}$ LHC [110]. The corresponding limits for the top partner mass are always satisfied for the choice of $v_{R}$ in our model. We note that the signal cross section decreases when the vectorlike fermion mass is 


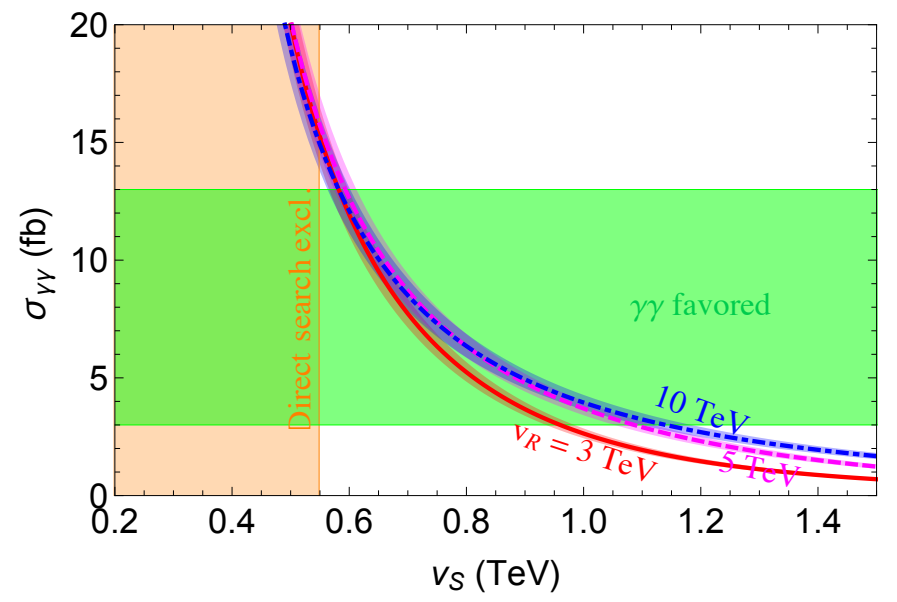

Figure 3. The LR seesaw parameter space fitting the $\sqrt{s}=13 \mathrm{TeV}$ diphoton excess, as a function of the singlet VEV $v_{S}$. The bands around each line for a given $v_{R}$ shows the uncertainties from parton distribution functions and $\alpha_{s}$ [122]. The horizontal (green) band shows the best-fit range to explain the diphoton excess at the $\sqrt{s}=13 \mathrm{TeV}$ LHC [1,2], whereas the vertical (orange) shaded region is ruled out from direct searches for heavy vectorlike quarks at $\sqrt{s}=8 \mathrm{TeV}$ LHC [110].

heavier, as expected. Furthermore, the mild dependence on the $\mathrm{RH}$ scale $v_{R}$ in some region of the parameter space is mainly from the mixing of SM quarks and their heavy partners.

In order to see whether the allowed parameter space shown in figure 3 is consistent with the upper limits on signal cross sections in other channels from the publicly available $\sqrt{s}=8 \mathrm{TeV}$ data, we evaluate the coresponding production cross section $\sigma(g g \rightarrow S)^{8 \mathrm{TeV}}=$ $\sigma_{0}^{8 \mathrm{TeV}} r$, where $\sigma_{0}^{8 \mathrm{TeV}}=156.8 \mathrm{fb}[120]$ and $r$ is the scaling factor defined in eq. (3.1). Using eqs. (3.8)-(3.12) and (3.13), we calculate the corresponding signal cross sections $\sigma_{X Y}^{8 \mathrm{TeV}} \equiv \sigma(g g \rightarrow S)^{8 \mathrm{TeV}} \times \mathrm{BR}(S \rightarrow X Y)$, where $X Y=t \bar{t}, g g, \gamma \gamma, \gamma Z$ and $Z Z$, respectively. These values are to be compared with the $95 \%$ CL upper limits on the cross sections obtained with the $\sqrt{s}=8 \mathrm{TeV}$ LHC data [123-129], namely,

$$
\sigma_{t \bar{t}}<450 \mathrm{fb}, \quad \sigma_{g g}<2.5 \mathrm{pb}, \quad \sigma_{\gamma \gamma}<1.5 \mathrm{fb}, \quad \sigma_{\gamma Z}<4 \mathrm{fb}, \quad \sigma_{Z Z}<12 \mathrm{fb} .
$$

In figure 4 , we compare the parameter space in the $\left(v_{S}, v_{R}\right)$ plane as preferred by the $\sqrt{s}=13 \mathrm{TeV}$ diphoton excess (green shaded region) with the $\sqrt{s}=8 \mathrm{TeV}$ exclusion limits given by eq. (3.14). We find that the most stringent constraint comes from the $\sqrt{s}=8 \mathrm{TeV}$ diphoton search, as shown by the red shaded region in figure 4 , which disfavors part of the $\sqrt{s}=13 \mathrm{TeV}$ diphoton excess region. The $\gamma Z$ and dijet constraints also rule out the low vectorlike fermion mass region, as shown by the magenta and blue shaded regions, respectively. The $t \bar{t}$ and $Z Z$ limits are much weaker in this model and only rule out the very low $v_{R}$ and $M_{F}$ values (not shown in this plot). The direct search limit [110] rules out the parameter space with $M_{B}<575 \mathrm{GeV}$, as shown by the orange shaded region. In any case, we find that $M_{F}(F \neq T)$ values between about $700-1150 \mathrm{GeV}$ are still compatible with the existing constraints and can explain the observed diphoton excess in this model.

It is interesting to note that for a fixed value of the singlet VEV $v_{S}$, there exists an upper limit on the RH scale $v_{R}$. This is shown in figure 5 where the different contours 


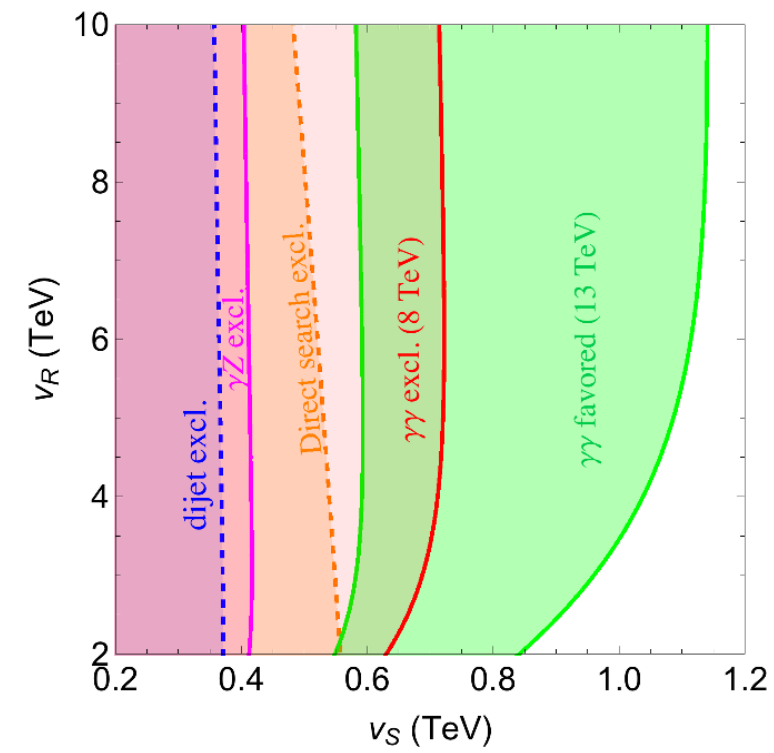

Figure 4. The LR seesaw parameter space fitting the $\sqrt{s}=13 \mathrm{TeV}$ diphoton excess (green), compared with the various exclusion limits from $\sqrt{s}=8 \mathrm{TeV}$ LHC data.

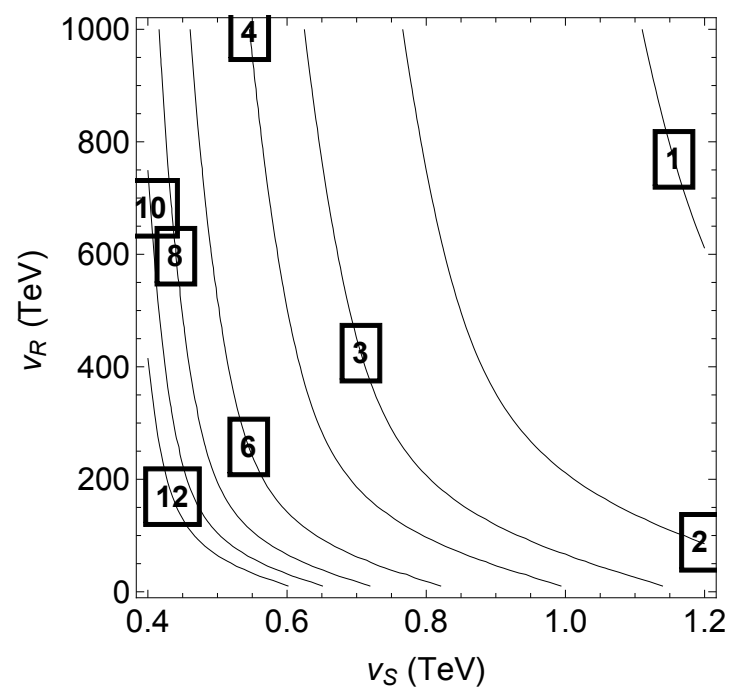

Figure 5. Contours of fixed $\sigma_{\gamma \gamma}$ (in fb) in the $\left(v_{S}, v_{R}\right)$ plane.

show the diphoton signal cross section $\sigma_{\gamma \gamma}$ in fb at $\sqrt{s}=13 \mathrm{TeV}$ LHC. We find that for $v_{S}=1 \mathrm{TeV}, v_{R}$ must be smaller than $65 \mathrm{TeV}$ to satisfy the observed value of $\sigma_{\gamma \gamma}$. For higher values of $v_{S}$, the upper limit on $v_{R}$ becomes stronger. This provides another potential way to distinguish this model from other explanations of the diphoton excess.

For the testability of this model in the ongoing run of the $\sqrt{s}=13 \mathrm{TeV}$ LHC in near future, we choose two optimum sets of parameters with the RH scale $v_{R}=3 \mathrm{TeV}$ and $5 \mathrm{TeV}$ respectively, for which the total production cross section, total decay width, the dominant fractional width and branching ratios of $S$ are listed in table 1. 


\begin{tabular}{|c|c|c|}
\hline & $v_{R}=3 \mathrm{TeV}$ & $v_{R}=5 \mathrm{TeV}$ \\
\hline \hline$f_{F}$ (input) & 1 & 1 \\
\hline$v_{S}[\mathrm{GeV}]$ (input) & 800 & 1000 \\
\hline$\sigma(g g \rightarrow S)[\mathrm{pb}]$ & 1.61 & 0.95 \\
\hline$\Gamma_{\text {total }}(S)[\mathrm{GeV}]$ & 0.21 & 0.071 \\
\hline \hline \multicolumn{2}{|c|}{ signal cross section $[\mathrm{fb}]$} \\
\hline$t \bar{t}$ & 423 & 122 \\
\hline$g g$ & 1173 & 825 \\
\hline$\gamma \gamma$ & 5.3 & 3.7 \\
\hline$\gamma Z$ & 3.2 & 2.3 \\
\hline$Z Z$ & 0.48 & 0.34 \\
\hline
\end{tabular}

Table 1. Input parameters $\left(f_{F}\right.$ and $\left.v_{S}\right)$ for $v_{R}=3 \mathrm{TeV}$ and $5 \mathrm{TeV}$, the total production cross section and decay width of the scalar $S$, and the signal cross sections in various relevant channels at the $\sqrt{s}=13 \mathrm{TeV}$ LHC in the minimal LR seesaw model.

\section{High-scale validity}

Due to the presence of a large number of extra fermions in our model and their $\mathcal{O}(1)$ Yukawa couplings with the singlet scalar field $S$, it is expected that the singlet scalar self-coupling $\lambda_{S}$ would become negative well before the Planck scale, thus potentially destabilizing the singlet vacuum. This would mean that our TeV-scale model provides only an effective description of the $750 \mathrm{GeV}$ diphoton excess and must be augmented by some other new physics for its ultraviolet (UV)-completion. In order to find this cut-off scale, we write down the one-loop renormalization group (RG) equation for the scalar self-coupling $\lambda_{S}$, as well as for other relevant couplings affecting the evolution of $\lambda_{S}$ above the scale $v_{S}:{ }^{2}$

$$
\begin{aligned}
16 \pi^{2} \frac{d g_{s}}{d t} & =-3 g_{s}^{3} \\
16 \pi^{2} \frac{d g_{B L}}{d t} & =\frac{41}{2} g_{B L}^{3} \\
16 \pi^{2} \frac{d \lambda_{S}}{d t} & =72 \lambda_{S}^{2}+24 \lambda_{S}\left[3\left(f_{U}^{2}+f_{D}^{2}\right)+f_{E}^{2}\right]-6\left[3\left(f_{U}^{4}+f_{D}^{4}\right)+f_{E}^{4}\right] \\
16 \pi^{2} \frac{d f_{U}}{d t} & =21 f_{U}^{3}-8 g_{s}^{2} f_{U}-\frac{8}{3} g_{B L}^{2} f_{U} \\
16 \pi^{2} \frac{d f_{D}}{d t} & =21 f_{D}^{3}-8 g_{s}^{2} f_{D}-\frac{2}{3} g_{B L}^{2} f_{D}, \\
16 \pi^{2} \frac{d f_{E}}{d t} & =9 f_{E}^{3}-2 g_{B L}^{2} f_{E}
\end{aligned}
$$

where $t \equiv \log (\mu)$ is the renormalization scale, and for simplicity, we have assumed a common Yukawa coupling $f_{F}$ (with $F=U, D, E$ ) for each of the three generations of the vectorlike fermions. We have further assumed the coupling $\lambda_{3}$ in eq. (2.1) to be very small, so that it does not affect the RG evolution of other couplings and the electroweak vacuum stability

\footnotetext{
${ }^{2}$ See also refs. [130-132] for similar RG analyses in presence of vectorlike fermions.
} 

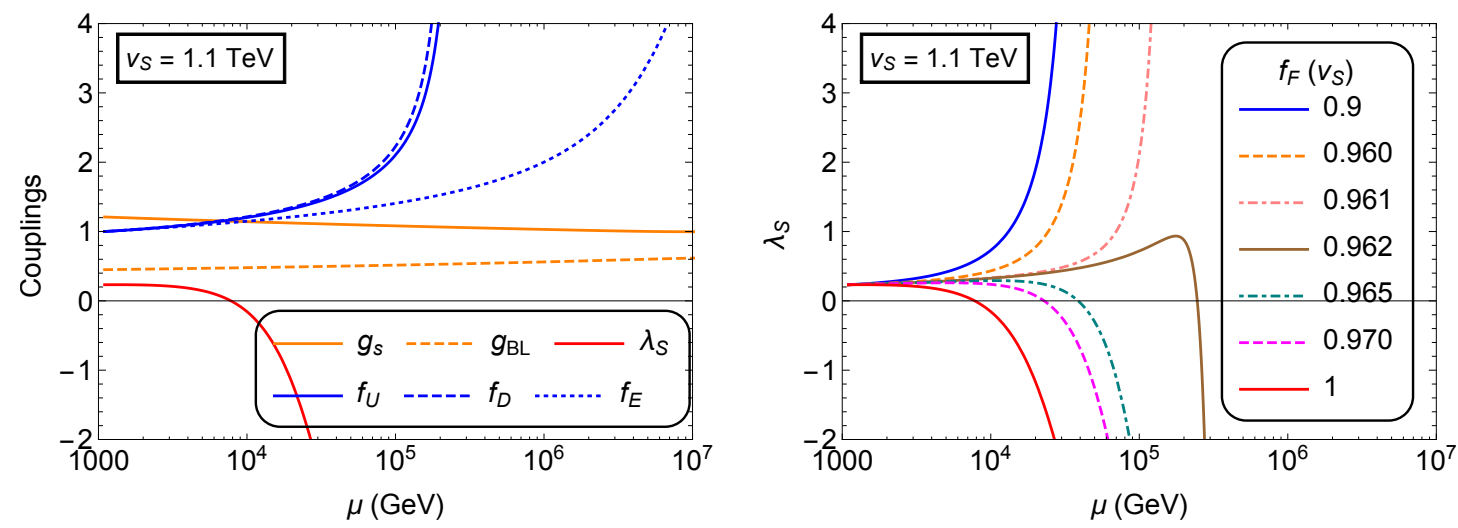

Figure 6. (Left) The one-loop RG evolution of the couplings given by eqs. (4.1)-(4.6) for a typical choice of the singlet VEV $v_{S}=1.1 \mathrm{TeV}$ and with the initial condition $f_{U}=f_{D}=f_{E}=0.98$ at $\mu=v_{S}$. (Right) The dependence of the $\lambda_{S}$ running on the initial conditions for $f_{U, D, E}\left(v_{S}\right)$.

analysis, as given in ref. [108]. For a given value of the singlet VEV $v_{S}$, the initial value of $\lambda_{S}\left(v_{S}\right)$ is fixed by eq. (2.2) which, for small $\lambda_{3}$, reads $\lambda_{S}\left(v_{S}\right) \simeq \frac{m_{S}^{2}}{2 v_{S}^{2}}$ with $m_{S}=750 \mathrm{GeV}$. For illustration, we choose $v_{S}=1.1 \mathrm{TeV}$ which is close to the maximum value allowed by the diphoton excess [cf. figure 4]. For the vectorlike fermion couplings $f_{F}\left(v_{S}\right)=1$, we find a cut-off scale around $10 \mathrm{TeV}$, as shown in figure 6 (left). For larger initial values of the Yukawa couplings $f_{F}$, the cut-off scale will be even lower, as is evident from eq. (4.3). For slightly smaller values of $f_{F}$, the cut-off scale can be pushed up to about $300 \mathrm{TeV}$, as can be seen from figure 6 (right). But as we further lower the initial value of $f_{F}$, the positive contribution to the r.h.s. of eq. (4.3) overtakes the negative one, thus resulting in non-perturbative values of $\lambda_{S}$. In any case, the diphoton signal rate also decreases with $f_{F}$ [cf. eq. (3.1)], while the direct search constraints on $M_{F} \simeq f_{F} v_{S}$ require a larger $v_{S}$. Thus, it turns out that we must have $f_{F}\left(v_{S}\right) \gtrsim 0.5$ to allow some model parameter space satisfying the diphoton excess.

\section{Discussions}

In general vectorlike fermion and (pseudo)scalar interpretations of the diphoton events recently observed at $750 \mathrm{GeV}$, the Yukawa couplings of vectorlike quarks to the scalar resonance tend to be very large, or the electric charges of the heavy fermions are exotically large. It is preferable in the LR seesaw model that these problems are avoided by the naturally large number of heavy partners for the seesaw generation of SM fermion masses. We emphasize here that while the main features of the diphoton explanation in this model are quite similar to earlier works which introduced a SM-singlet scalar and vectorlike fermions, the specific nature of our model constrained by the strong $C P$ requirement, i.e. seesaw quark masses, leads to some differences. In particular, our minimal LR interpretation of the diphoton excess implies the following features which could be tested by the upcoming LHC data and future precision measurements: 
(i) As the gluon fusion production for $S$ goes down like $M_{F}^{-2}$, the observation of diphoton events with cross section at the level of few fb implies the existence of heavy fermions at the $\mathrm{TeV}$ scale.

(ii) In the minimal LR seesaw model, for heavy partners at the scale of $1 \mathrm{TeV}$, the $\mathrm{RH}$ scale lies naturally at the few $\mathrm{TeV}$ scale. In such scenarios we expect a heavy $W_{R}$ nearby which should be accessible at the $\sqrt{s}=13 \mathrm{TeV}$ LHC. ${ }^{3}$

(iii) The decay of $S$ in the LR model is suppressed either by small mixing parameters or the loop factors, and thus it would be a narrow resonance and could be easily tested by the upcoming LHC data.

(iv) A large portion of the $S$ decay width is into top quark pairs, with a branching ratio varying from a few percent to half or even larger (subject to the top quark mixing and other parameters). Thus, the $t \bar{t}$ observations could not only test the minimal LR model but also be used to probe the $t-T$ mixing which is essential to search for new physics in the top quark sector.

(v) In the minimal scenario of the LR seesaw model, the singlet $S$ decouples from the doublets for gauge symmetry breaking. In such cases the stability analysis of the scalar potential is the same as in [108]. In a large parameter space the SM vacuum is stable up to the GUT scale or even the Planck scale.

(vi) The presence of the new scalar $S$ and the vectorlike fermions has a potential impact on the precision electroweak observables [142]. However, with the small $t-T$ mixing angle $\sin \alpha_{L}^{t}$ as in our case [cf. eq. (3.4)], these constraints are easily satisfied for the range of top-partner masses considered here. In future, a better measurement of the top-quark decay width which has currently a large uncertainty of $\mathcal{O}(1 \mathrm{GeV})[143,144]$ could be another way to test our hypothesis.

(vii) Due to the presence of a large number of vectorlike fermions in the model, the scalar self-coupling becomes negative at around 10-100 TeV (see section 4), thus signaling the onset of some other new physics for its UV-completion. This new physics could be connected with the appearance of an expanded gauge group, e.g. $\mathrm{SU}(2)_{L} \times \mathrm{SU}(2)_{R} \times \mathrm{U}(1)_{B-L, L} \times \mathrm{U}(1)_{B-L, R}$ or $\mathrm{SU}(5) \times \mathrm{SU}(5)$. These topics are currently under investigation.

\section{Conclusion}

We have presented a left-right quark seesaw model with $\mathrm{TeV}$-scale vectorlike fermions and a singlet scalar, where the singlet scalar responsible for the mass of the vectorlike fermions can be identified as the new $750 \mathrm{GeV}$ resonance indicated by the early $\sqrt{s}=13 \mathrm{TeV}$ LHC data. This model was originally proposed to solve the strong CP problem without the axion. We have discussed various ways this hypothesis can be tested in near future as more data is amassed in the run II of the LHC.

\footnotetext{
${ }^{3}$ The recent diboson excess $[133,134]$ suggests a $W_{R}$ with mass around $1.9-2 \mathrm{TeV}$ and $g_{R}=0.4-0.5$ (see e.g. [135-141]). This scenario still remains to be tested by the $\sqrt{s}=13 \mathrm{TeV}$ data.
} 


\section{Acknowledgments}

Y.Z. thanks Julian Heeck for inspiring discussions on the singlet in LR seesaw model. The work of P.S.B.D is supported by the DFG with grant RO 2516/5-1. The work of R.N.M. is supported in part by the US National Science Foundation Grant No. PHY-1315155. Y.Z. would like to thank the IISN and Belgian Science Policy (IAP VII/37) for support.

\section{A Exact formulae for the $\gamma Z$ and $Z Z$ channel}

The exact formulae for the loop-induced $\gamma Z$ and $Z Z$ decay channels of $S$ are respectively

$$
\begin{aligned}
& \Gamma_{\gamma Z}=\frac{\alpha^{2} M_{S}^{3}}{32 \pi^{3} s_{w}^{2} c_{w}^{2}}\left(1-\frac{M_{Z}^{2}}{M_{S}^{2}}\right)^{3}\left|\sum_{i=t, P, N, E} \frac{f_{i}}{M_{i}} N_{C i} Q_{i}\left(I_{3 i}-2 Q_{i} s_{w}^{2}\right) A_{1 / 2}\left(\tau_{i}^{-1}, \lambda_{i}^{-1}\right)\right|^{2}, \\
& \Gamma_{Z Z}=\frac{\alpha^{2} M_{S}^{3}}{256 \pi^{3} s_{w}^{4} c_{w}^{4}}\left(1-\frac{4 M_{Z}^{2}}{M_{S}^{2}}\right)^{1 / 2}\left|\sum_{i=t, P, N, E} \frac{f_{i}}{M_{i}} N_{C i}\left(\frac{1}{2} I_{3 i}-Q_{i} s_{w}^{2}\right)^{2} A_{Z Z}\left(\tau_{i}, \lambda_{i}\right)\right|^{2} .
\end{aligned}
$$

The loop function $A_{1 / 2}(\tau, \lambda)$ is given by [121]

$$
\begin{aligned}
A_{1 / 2}(\tau, \lambda)= & \frac{\tau \lambda}{2(\tau-\lambda)}+\frac{\tau^{2} \lambda^{2}}{2(\tau-\lambda)^{2}}\left[f\left(\tau^{-1}\right)-f\left(\lambda^{-1}\right)\right]+\frac{\tau^{2} \lambda}{(\tau-\lambda)^{2}}\left[\left(g\left(\tau^{-1}\right)-g\left(\lambda^{-1}\right)\right]\right. \\
& +\frac{\tau \lambda}{2(\tau-\lambda}\left[f\left(\tau^{-1}\right)-f\left(\lambda^{-1}\right)\right]
\end{aligned}
$$

where the function $f(x)$ is defined in eq. (3.3) and $g(x)$ is defined as

$$
g(x)= \begin{cases}\frac{\sqrt{1-1 / x}}{2}\left[\log \left(\frac{1+\sqrt{1-1 / x}}{1-\sqrt{1-1 / x}}\right)-i \pi\right] & \text { if } x<1 \\ \sqrt{1 / x-1} \arcsin (\sqrt{x}) & \text { if } x \geq 1 .\end{cases}
$$

For the $Z Z$ channel,

$$
\begin{aligned}
A_{Z Z}^{2}(\tau, \lambda)= & \frac{2}{\tau^{2}}\left[2+2\left|f_{1}\right|\left(\frac{1-\tau}{\tau}+\frac{1-\beta^{4}}{4 \beta^{2}}\right)-\left|f_{2}\right| \frac{\left(1-\beta^{2}\right)}{\beta^{2}}\right. \\
& +\left|f_{1}\right|^{2}\left\{3 \tau^{2}-8 \beta^{2}(\tau-1) \tau+2 \beta^{4}\left(5 \tau^{2}-16 \tau+8\right)+8 \beta^{6}(\tau-1) \tau+3 \beta^{8} \tau^{2}\right\} \\
& \left.+\left|f_{2}\right|^{2} \frac{3\left(1-\beta^{2}\right)^{2}}{8 \beta^{4}}+\frac{\left|f_{1} f_{2}\right|}{8 \beta^{4} \tau}\left(1-\beta^{2}\right)\left(-3 \tau+4 \beta^{2}(\tau-1)+3 \beta^{4} \tau\right)\right]
\end{aligned}
$$

where $\tau=M_{S}^{2} / 4 M_{F}^{2}, \lambda=M_{Z}^{2} / 4 M_{F}^{2}, \beta=\sqrt{1-4 M_{Z}^{2} / M_{S}^{2}}$ and

$$
\begin{aligned}
f_{1}(\tau, \lambda) & =-\int_{0}^{1} d x \int_{0}^{1-x} d y \frac{4 \tau}{1-4[x(1-x)+y(1-y)] \lambda-4 x y(\tau-2 \lambda)} \\
f_{2}(\tau, \lambda) & =2 f_{3}(\tau)-2 f_{3}(\lambda) \\
f_{3}(\tau) & = \begin{cases}-2+2 \sqrt{\frac{1}{\tau}-1} \arcsin (\sqrt{\tau}) & (\text { for } \tau \leq 1) \\
-2+i \pi \sqrt{1-1 / \tau}+\sqrt{1-1 / \tau} \tanh ^{-1}\left(\frac{2 \sqrt{(\tau-1) \tau}}{2 \tau-1}\right) & (\text { for } \tau>1) .\end{cases}
\end{aligned}
$$


In the limit of $\beta \rightarrow 1$, only the function $f_{1}(\tau)$ contributes and the loop function $A_{Z Z}(\tau, \lambda) \rightarrow$ $A_{1 / 2}(\tau)$, thus yielding eqs. (3.11) and (3.12).

Open Access. This article is distributed under the terms of the Creative Commons Attribution License (CC-BY 4.0), which permits any use, distribution and reproduction in any medium, provided the original author(s) and source are credited.

\section{References}

[1] CMS collaboration, Search for new physics in high mass diphoton events in proton-proton collisions at $\sqrt{s}=13 \mathrm{TeV}$, CMS-PAS-EXO-15-004 (2015).

[2] ATLAS collaboration, Search for resonances decaying to photon pairs in $3.2 \mathrm{fb}^{-1}$ of pp collisions at $\sqrt{s}=13 \mathrm{TeV}$ with the ATLAS detector, ATLAS-CONF-2015-081 (2015).

[3] K. Harigaya and Y. Nomura, Composite Models for the $750 \mathrm{GeV}$ Diphoton Excess, Phys. Lett. B 754 (2016) 151 [arXiv: 1512.04850] [INSPIRE].

[4] Y. Mambrini, G. Arcadi and A. Djouadi, The LHC diphoton resonance and dark matter, arXiv:1512.04913 [INSPIRE].

[5] M. Backovic, A. Mariotti and D. Redigolo, Di-photon excess illuminates Dark Matter, arXiv: 1512.04917 [INSPIRE].

[6] A. Angelescu, A. Djouadi and G. Moreau, Scenarii for interpretations of the LHC diphoton excess: two Higgs doublets and vector-like quarks and leptons, arXiv:1512.04921 [INSPIRE].

[7] Y. Nakai, R. Sato and K. Tobioka, Footprints of New Strong Dynamics via Anomaly, arXiv:1512.04924 [INSPIRE].

[8] S. Knapen, T. Melia, M. Papucci and K. Zurek, Rays of light from the LHC, arXiv: 1512.04928 [INSPIRE].

[9] D. Buttazzo, A. Greljo and D. Marzocca, Knocking on New Physics' door with a Scalar Resonance, arXiv: 1512.04929 [INSPIRE].

[10] A. Pilaftsis, Diphoton Signatures from Heavy Axion Decays at the CERN Large Hadron Collider, Phys. Rev. D 93 (2016) 015017 [arXiv:1512.04931] [INSPIRE].

[11] R. Franceschini et al., What is the gamma gamma resonance at $750 \mathrm{GeV}$ ?, arXiv: 1512.04933 [INSPIRE].

[12] S. Di Chiara, L. Marzola and M. Raidal, First interpretation of the $750 \mathrm{GeV}$ di-photon resonance at the $L H C$, arXiv: 1512.04939 [INSPIRE].

[13] T. Higaki, K.S. Jeong, N. Kitajima and F. Takahashi, The QCD Axion from Aligned Axions and Diphoton Excess, Phys. Lett. B 755 (2016) 13 [arXiv:1512.05295] [INSPIRE].

[14] S.D. McDermott, P. Meade and H. Ramani, Singlet Scalar Resonances and the Diphoton Excess, arXiv:1512.05326 [INSPIRE].

[15] J. Ellis, S.A.R. Ellis, J. Quevillon, V. Sanz and T. You, On the Interpretation of a Possible $\sim 750$ GeV Particle Decaying into $\gamma \gamma$, arXiv:1512.05327 [INSPIRE].

[16] M. Low, A. Tesi and L.-T. Wang, A pseudoscalar decaying to photon pairs in the early LHC Run 2 data, arXiv:1512.05328 [INSPIRE]. 
[17] B. Bellazzini, R. Franceschini, F. Sala and J. Serra, Goldstones in Diphotons, arXiv: 1512.05330 [INSPIRE].

[18] R.S. Gupta, S. Jäger, Y. Kats, G. Perez and E. Stamou, Interpreting a 750 GeV Diphoton Resonance, arXiv: 1512.05332 [INSPIRE].

[19] C. Petersson and R. Torre, The $750 \mathrm{GeV}$ diphoton excess from the goldstino superpartner, arXiv: 1512.05333 [INSPIRE].

[20] E. Molinaro, F. Sannino and N. Vignaroli, Minimal Composite Dynamics versus Axion Origin of the Diphoton excess, arXiv:1512.05334 [INSPIRE].

[21] B. Dutta, Y. Gao, T. Ghosh, I. Gogoladze and T. Li, Interpretation of the diphoton excess at $C M S$ and $A T L A S$, arXiv:1512.05439 [INSPIRE].

[22] Q.-H. Cao, Y. Liu, K.-P. Xie, B. Yan and D.-M. Zhang, A Boost Test of Anomalous Diphoton Resonance at the LHC, arXiv:1512.05542 [INSPIRE].

[23] S. Matsuzaki and K. Yamawaki, $750 \mathrm{GeV}$ Diphoton Signal from One-Family Walking Technipion, arXiv: 1512.05564 [INSPIRE].

[24] A. Kobakhidze, F. Wang, L. Wu, J.M. Yang and M. Zhang, LHC 750 GeV diphoton resonance explained as a heavy scalar in top-seesaw model, arXiv:1512.05585 [INSPIRE].

[25] R. Martinez, F. Ochoa and C.F. Sierra, Diphoton decay for a $750 \mathrm{GeV}$ scalar boson in an $\mathrm{U}(1)^{\prime}$ model, arXiv: 1512.05617 [INSPIRE].

[26] P. Cox, A.D. Medina, T.S. Ray and A. Spray, Diphoton Excess at $750 \mathrm{GeV}$ from a Radion in the Bulk-Higgs Scenario, arXiv:1512.05618 [INSPIRE].

[27] D. Becirevic, E. Bertuzzo, O. Sumensari and R.Z. Funchal, Can the new resonance at LHC be a CP-Odd Higgs boson?, arXiv:1512.05623 [INSPIRE].

[28] J.M. No, V. Sanz and J. Setford, See-Saw Composite Higgses at the LHC: Linking Naturalness to the $750 \mathrm{GeV}$ Di-Photon Resonance, arXiv: 1512.05700 [INSPIRE].

[29] S.V. Demidov and D.S. Gorbunov, On sgoldstino interpretation of the diphoton excess, arXiv: 1512.05723 [INSPIRE].

[30] W. Chao, R. Huo and J.-H. Yu, The Minimal Scalar-Stealth Top Interpretation of the Diphoton Excess, arXiv:1512.05738 [INSPIRE].

[31] S. Fichet, G. von Gersdorff and C. Royon, Scattering Light by Light at $750 \mathrm{GeV}$ at the LHC, arXiv: 1512.05751 [INSPIRE].

[32] D. Curtin and C.B. Verhaaren, Quirky Explanations for the Diphoton Excess, arXiv: 1512.05753 [INSPIRE].

[33] L. Bian, N. Chen, D. Liu and J. Shu, A hidden confining world on the $750 \mathrm{GeV}$ diphoton excess, arXiv: 1512.05759 [INSPIRE].

[34] J. Chakrabortty, A. Choudhury, P. Ghosh, S. Mondal and T. Srivastava, Di-photon resonance around $750 \mathrm{GeV}$ : shedding light on the theory underneath, arXiv:1512.05767 [INSPIRE].

[35] A. Ahmed, B.M. Dillon, B. Grzadkowski, J.F. Gunion and Y. Jiang, Higgs-radion interpretation of $750 \mathrm{GeV}$ di-photon excess at the LHC, arXiv:1512.05771 [INSPIRE].

[36] P. Agrawal, J. Fan, B. Heidenreich, M. Reece and M. Strassler, Experimental Considerations Motivated by the Diphoton Excess at the LHC, arXiv:1512.05775 [INSPIRE]. 
[37] C. Csáki, J. Hubisz and J. Terning, Minimal model of a diphoton resonance: Production without gluon couplings, Phys. Rev. D 93 (2016) 035002 [arXiv:1512.05776] [INSPIRE].

[38] A. Falkowski, O. Slone and T. Volansky, Phenomenology of a $750 \mathrm{GeV}$ Singlet, arXiv: 1512.05777 [INSPIRE].

[39] D. Aloni, K. Blum, A. Dery, A. Efrati and Y. Nir, On a possible large width $750 \mathrm{GeV}$ diphoton resonance at ATLAS and CMS, arXiv:1512.05778 [INSPIRE].

[40] Y. Bai, J. Berger and R. Lu, A 750 GeV Dark Pion: Cousin of a Dark G-parity-odd WIMP, arXiv: 1512.05779 [INSPIRE].

[41] E. Gabrielli, K. Kannike, B. Mele, M. Raidal, C. Spethmann and H. Veermäe, A SUSY Inspired Simplified Model for the $750 \mathrm{GeV}$ Diphoton Excess, arXiv:1512.05961 [INSPIRE].

[42] R. Benbrik, C.-H. Chen and T. Nomura, Higgs singlet as a diphoton resonance in a vector-like quark model, arXiv:1512.06028 [INSPIRE].

[43] J.S. Kim, J. Reuter, K. Rolbiecki and R.R. de Austri, A resonance without resonance: scrutinizing the diphoton excess at $750 \mathrm{GeV}$, arXiv: 1512.06083 [INSPIRE].

[44] A. Alves, A.G. Dias and K. Sinha, The $750 \mathrm{GeV}$ S-cion: Where else should we look for it?, arXiv: 1512.06091 [INSPIRE].

[45] E. Megias, O. Pujolàs and M. Quirós, On dilatons and the LHC diphoton excess, arXiv: 1512.06106 [INSPIRE].

[46] L.M. Carpenter, R. Colburn and J. Goodman, Supersoft SUSY Models and the $750 \mathrm{GeV}$ Diphoton Excess, Beyond Effective Operators, arXiv:1512.06107 [INSPIRE].

[47] J. Bernon and C. Smith, Could the width of the diphoton anomaly signal a three-body decay?, arXiv:1512.06113 [INSPIRE].

[48] W. Chao, Symmetries Behind the 750 GeV Diphoton Excess, arXiv:1512.06297 [INSPIRE].

[49] M.T. Arun and P. Saha, Gravitons in multiply warped scenarios - at $750 \mathrm{GeV}$ and beyond, arXiv: 1512.06335 [INSPIRE].

[50] C. Han, H.M. Lee, M. Park and V. Sanz, The diphoton resonance as a gravity mediator of dark matter, arXiv:1512.06376 [INSPIRE].

[51] S. Chang, A Simple U(1) Gauge Theory Explanation of the Diphoton Excess, arXiv:1512.06426 [INSPIRE].

[52] I. Chakraborty and A. Kundu, Diphoton excess at $750 \mathrm{GeV}$ : Singlet scalars confront triviality, arXiv:1512.06508 [INSPIRE].

[53] R. Ding, L. Huang, T. Li and B. Zhu, Interpreting 750 GeV Diphoton Excess with R-parity Violation Supersymmetry, arXiv: 1512.06560 [INSPIRE].

[54] H. Han, S. Wang and S. Zheng, Scalar Explanation of Diphoton Excess at LHC, arXiv: 1512.06562 [INSPIRE].

[55] X.-F. Han and L. Wang, Implication of the $750 \mathrm{GeV}$ diphoton resonance on two-Higgs-doublet model and its extensions with Higgs field, arXiv:1512.06587 [INSPIRE].

[56] M.-x. Luo, K. Wang, T. Xu, L. Zhang and G. Zhu, Squarkonium/Diquarkonium and the Di-photon Excess, arXiv:1512.06670 [INSPIRE].

[57] J. Chang, K. Cheung and C.-T. Lu, Interpreting the $750 \mathrm{GeV}$ Di-photon Resonance using photon-jets in Hidden-Valley-like models, arXiv:1512.06671 [INSPIRE]. 
[58] D. Bardhan, D. Bhatia, A. Chakraborty, U. Maitra, S. Raychaudhuri and T. Samui, Radion Candidate for the LHC Diphoton Resonance, arXiv:1512.06674 [INSPIRE].

[59] T.-F. Feng, X.-Q. Li, H.-B. Zhang and S.-M. Zhao, The LHC $750 \mathrm{GeV}$ diphoton excess in supersymmetry with gauged baryon and lepton numbers, arXiv:1512.06696 [INSPIRE].

[60] O. Antipin, M. Mojaza and F. Sannino, A natural Coleman-Weinberg theory explains the diphoton excess, arXiv:1512.06708 [INSPIRE].

[61] F. Wang, L. Wu, J.M. Yang and M. Zhang, 750 GeV Diphoton Resonance, 125 GeV Higgs and Muon g-2 Anomaly in Deflected Anomaly Mediation SUSY Breaking Scenario, arXiv: 1512.06715 [INSPIRE].

[62] J. Cao, C. Han, L. Shang, W. Su, J.M. Yang and Y. Zhang, Interpreting the $750 \mathrm{GeV}$ diphoton excess by the singlet extension of the Manohar-Wise Model, arXiv:1512.06728 [INSPIRE].

[63] F.P. Huang, C.S. Li, Z.L. Liu and Y. Wang, 750 GeV Diphoton Excess from Cascade Decay, arXiv: 1512.06732 [INSPIRE].

[64] W. Liao and H.-q. Zheng, Scalar resonance at $750 \mathrm{GeV}$ as composite of heavy vector-like fermions, arXiv:1512.06741 [INSPIRE].

[65] J.J. Heckman, $750 \mathrm{GeV}$ Diphotons from a D3-brane, arXiv:1512.06773 [INSPIRE].

[66] X.-J. Bi, Q.-F. Xiang, P.-F. Yin and Z.-H. Yu, The $750 \mathrm{GeV}$ diphoton excess at the LHC and dark matter constraints, arXiv:1512.06787 [INSPIRE].

[67] J.S. Kim, K. Rolbiecki and R.R. de Austri, Model-independent combination of diphoton constraints at $750 \mathrm{GeV}$, arXiv: 1512.06797 [INSPIRE].

[68] L. Berthier, J.M. Cline, W. Shepherd and M. Trott, Effective interpretations of a diphoton excess, arXiv: 1512.06799 [INSPIRE].

[69] W.S. Cho et al., The $750 \mathrm{GeV}$ Diphoton Excess May Not Imply a $750 \mathrm{GeV}$ Resonance, arXiv: 1512.06824 [INSPIRE].

[70] J.M. Cline and Z. Liu, LHC diphotons from electroweakly pair-produced composite pseudoscalars, arXiv:1512.06827 [INSPIRE].

[71] M. Bauer and M. Neubert, Flavor Anomalies, the Diphoton Excess and a Dark Matter Candidate, arXiv:1512.06828 [INSPIRE].

[72] M. Chala, M. Duerr, F. Kahlhoefer and K. Schmidt-Hoberg, Tricking Landau-Yang: How to obtain the diphoton excess from a vector resonance, Phys. Lett. B 755 (2016) 145 [arXiv: 1512.06833] [INSPIRE].

[73] D. Barducci, A. Goudelis, S. Kulkarni and D. Sengupta, One jet to rule them all: monojet constraints and invisible decays of a $750 \mathrm{GeV}$ diphoton resonance, arXiv:1512.06842 [INSPIRE].

[74] S.M. Boucenna, S. Morisi and A. Vicente, The LHC diphoton resonance from gauge symmetry, arXiv:1512.06878 [INSPIRE].

[75] C.W. Murphy, Vector Leptoquarks and the $750 \mathrm{GeV}$ Diphoton Resonance at the LHC, arXiv: 1512.06976 [INSPIRE].

[76] A.E.C. Hernández and I. Nisandzic, LHC diphoton $750 \mathrm{GeV}$ resonance as an indication of $\mathrm{SU}(3)_{c} \times \mathrm{SU}(3)_{L} \times \mathrm{U}(1)_{X}$ gauge symmetry, arXiv:1512.07165 [INSPIRE]. 
[77] U.K. Dey, S. Mohanty and G. Tomar, 750 GeV resonance in the Dark Left-Right Model, arXiv: 1512.07212 [INSPIRE].

[78] G.M. Pelaggi, A. Strumia and E. Vigiani, Trinification can explain the di-photon and di-boson LHC anomalies, arXiv:1512.07225 [INSPIRE].

[79] J. de Blas, J. Santiago and R. Vega-Morales, New vector bosons and the diphoton excess, arXiv: 1512.07229 [INSPIRE].

[80] P.S.B. Dev and D. Teresi, Asymmetric Dark Matter in the Sun and the Diphoton Excess at the $L H C$, arXiv: 1512.07243 [INSPIRE].

[81] W.-C. Huang, Y.-L.S. Tsai and T.-C. Yuan, Gauged Two Higgs Doublet Model confronts the LHC $750 \mathrm{GeV}$ di-photon anomaly, arXiv: 1512.07268 [INSPIRE].

[82] S. Moretti and K. Yagyu, The $750 \mathrm{GeV}$ diphoton excess and its explanation in 2-Higgs Doublet Models with a real inert scalar multiplet, arXiv:1512.07462 [INSPIRE].

[83] K.M. Patel and P. Sharma, Interpreting $750 \mathrm{GeV}$ diphoton excess in $\mathrm{SU}(5)$ grand unified theory, arXiv: 1512.07468 [INSPIRE].

[84] M. Badziak, Interpreting the $750 \mathrm{GeV}$ diphoton excess in minimal extensions of Two-Higgs-Doublet models, arXiv:1512.07497 [INSPIRE].

[85] S. Chakraborty, A. Chakraborty and S. Raychaudhuri, Diphoton resonance at $750 \mathrm{GeV}$ in the broken MRSSM, arXiv:1512.07527 [INSPIRE].

[86] Q.-H. Cao, S.-L. Chen and P.-H. Gu, Strong CP Problem, Neutrino Masses and the $750 \mathrm{GeV}$ Diphoton Resonance, arXiv: 1512.07541 [INSPIRE].

[87] W. Altmannshofer, J. Galloway, S. Gori, A.L. Kagan, A. Martin and J. Zupan, On the $750 \mathrm{GeV}$ di-photon excess, arXiv:1512.07616 [INSPIRE].

[88] M. Cvetič, J. Halverson and P. Langacker, String Consistency, Heavy Exotics and the $750 \mathrm{GeV}$ Diphoton Excess at the LHC, arXiv:1512.07622 [INSPIRE].

[89] J. Gu and Z. Liu, Running after Diphoton, arXiv:1512.07624 [INSPIRE].

[90] B.C. Allanach, P.S.B. Dev, S.A. Renner and K. Sakurai, Di-photon Excess Explained by a Resonant Sneutrino in R-parity Violating Supersymmetry, arXiv:1512.07645 [INSPIRE].

[91] H. Davoudiasl and C. Zhang, A $750 \mathrm{GeV}$ Messenger of Dark Conformal Symmetry Breaking, arXiv: 1512.07672 [INSPIRE].

[92] N. Craig, P. Draper, C. Kilic and S. Thomas, How the $\gamma \gamma$ Resonance Stole Christmas, arXiv: 1512.07733 [INSPIRE].

[93] K. Das and S.K. Rai, The 750 GeV Diphoton excess in a U(1) hidden symmetry model, arXiv: 1512.07789 [INSPIRE].

[94] K. Cheung, P. Ko, J.S. Lee, J. Park and P.-Y. Tseng, A Higgcision study on the $750 \mathrm{GeV}$ Di-photon Resonance and $125 \mathrm{GeV}$ SM Higgs boson with the Higgs-Singlet Mixing, arXiv: 1512.07853 [INSPIRE].

[95] J. Liu, X.-P. Wang and W. Xue, LHC diphoton excess from colorful resonances, arXiv: 1512.07885 [INSPIRE].

[96] J. Zhang and S. Zhou, Electroweak Vacuum Stability and Diphoton Excess at $750 \mathrm{GeV}$, arXiv:1512.07889 [INSPIRE]. 
[97] J.A. Casas, J.R. Espinosa and J.M. Moreno, The 750 GeV Diphoton Excess as a First Light on Supersymmetry Breaking, arXiv:1512.07895 [INSPIRE].

[98] L.J. Hall, K. Harigaya and Y. Nomura, 750 GeV Diphotons: Implications for Supersymmetric Unification, arXiv:1512.07904 [INSPIRE].

[99] K.S. Babu and R.N. Mohapatra, CP Violation in Seesaw Models of Quark Masses, Phys. Rev. Lett. 62 (1989) 1079 [INSPIRE].

[100] K.S. Babu and R.N. Mohapatra, A Solution to the Strong CP Problem Without an Axion, Phys. Rev. D 41 (1990) 1286 [InSPIRE].

[101] Z.G. Berezhiani, The Weak Mixing Angles in Gauge Models with Horizontal Symmetry: A New Approach to Quark and Lepton Masses, Phys. Lett. B 129 (1983) 99 [inSPIRE].

[102] D. Chang and R.N. Mohapatra, Small and Calculable Dirac Neutrino Mass, Phys. Rev. Lett. 58 (1987) 1600 [INSPIRE].

[103] S. Rajpoot, See-saw masses for quarks and leptons in an ambidextrous electroweak interaction model, Mod. Phys. Lett. A 2 (1987) 307 [INSPIRE].

[104] A. Davidson and K.C. Wali, Universal Seesaw Mechanism?, Phys. Rev. Lett. 59 (1987) 393 [INSPIRE].

[105] J.C. Pati and A. Salam, Lepton Number as the Fourth Color, Phys. Rev. D 10 (1974) 275 [Erratum ibid. D 11 (1975) 703] [INSPIRE].

[106] R.N. Mohapatra and J.C. Pati, A Natural Left-Right Symmetry, Phys. Rev. D 11 (1975) 2558 [INSPIRE].

[107] G. Senjanović and R.N. Mohapatra, Exact Left-Right Symmetry and Spontaneous Violation of Parity, Phys. Rev. D 12 (1975) 1502 [InSPIRE].

[108] R.N. Mohapatra and Y. Zhang, TeV Scale Universal Seesaw, Vacuum Stability and Heavy Higgs, JHEP 06 (2014) 072 [arXiv:1401.6701] [INSPIRE].

[109] R.T. D'Agnolo and A. Hook, Finding the Strong CP problem at the LHC, arXiv: 1507.00336 [INSPIRE].

[110] ATLAS collaboration, Search for production of vector-like quark pairs and of four top quarks in the lepton-plus-jets final state in pp collisions at $\sqrt{s}=8 \mathrm{TeV}$ with the ATLAS detector, JHEP 08 (2015) 105 [arXiv: 1505. 04306] [INSPIRE].

[111] D. Guadagnoli, R.N. Mohapatra and I. Sung, Gauged Flavor Group with Left-Right Symmetry, JHEP 04 (2011) 093 [arXiv:1103.4170] [INSPIRE].

[112] F. del Aguila, M. Pérez-Victoria and J. Santiago, Observable contributions of new exotic quarks to quark mixing, JHEP 09 (2000) 011 [hep-ph/0007316] [INSPIRE].

[113] R. Kawasaki, T. Morozumi and H. Umeeda, Quark sector CP-violation of the universal seesaw model, Phys. Rev. D 88 (2013) 033019 [arXiv:1306.5080] [INSPIRE].

[114] F. del Aguila, J. de Blas and M. Pérez-Victoria, Electroweak Limits on General New Vector Bosons, JHEP 09 (2010) 033 [arXiv: 1005.3998] [INSPIRE].

[115] J.A. Aguilar-Saavedra, R. Benbrik, S. Heinemeyer and M. Pérez-Victoria, Handbook of vectorlike quarks: Mixing and single production, Phys. Rev. D 88 (2013) 094010 [arXiv: 1306.0572] [INSPIRE]. 
[116] S. Patra, Neutrinoless double beta decay process in left-right symmetric models without scalar bidoublet, Phys. Rev. D 87 (2013) 015002 [arXiv:1212.0612] [INSPIRE].

[117] N. Bonne and G. Moreau, Reproducing the Higgs boson data with vector-like quarks, Phys. Lett. B 717 (2012) 409 [arXiv: 1206.3360] [INSPIRE].

[118] G. Moreau, Constraining extra-fermion(s) from the Higgs boson data, Phys. Rev. D 87 (2013) 015027 [arXiv:1210 . 3977] [INSPIRE].

[119] A. Angelescu, A. Djouadi and G. Moreau, Vector-like top/bottom quark partners and Higgs physics at the LHC, arXiv:1510.07527 [INSPIRE].

[120] https://twiki.cern.ch/twiki/bin/view/LHCPhysics/CrossSections.

[121] A. Djouadi, The Anatomy of electro-weak symmetry breaking. I: The Higgs boson in the standard model, Phys. Rept. 457 (2008) 1 [hep-ph/0503172] [inSPIRE].

[122] https://twiki.cern.ch/twiki/bin/view/LHCPhysics/HiggsEuropeanStrategy2012\# SM_Higgs_production_cross_se_AN2.

[123] CMS collaboration, Search for resonant $t \bar{t}$ production in proton-proton collisions at $\sqrt{s}=8$ TeV, Phys. Rev. D 93 (2016) 012001 [arXiv:1506.03062] [inSPIRE].

[124] ATLAS collaboration, Search for new phenomena in the dijet mass distribution using $p-p$ collision data at $\sqrt{s}=8 \mathrm{TeV}$ with the ATLAS detector, Phys. Rev. D 91 (2015) 052007 [arXiv: 1407.1376] [INSPIRE].

[125] CMS collaboration, Search for Resonances Decaying to Dijet Final States at $\sqrt{s}=8 \mathrm{TeV}$ with Scouting Data, CMS-PAS-EXO-14-005 (2014).

[126] CMS collaboration, Search for new resonances in the diphoton final state in the range between 150 and $850 \mathrm{GeV}$ in pp collisions at $\sqrt{s}=8 \mathrm{TeV}$, CMS-PAS-HIG-14-006 (2014).

[127] ATLAS collaboration, Search for high-mass diphoton resonances in pp collisions at $\sqrt{s}=8 \mathrm{TeV}$ with the ATLAS detector, Phys. Rev. D 92 (2015) 032004 [arXiv: 1504.05511] [INSPIRE].

[128] ATLAS collaboration, Search for new resonances in $W \gamma$ and $Z_{\gamma}$ final states in $p p$ collisions at $\sqrt{s}=8 \mathrm{TeV}$ with the ATLAS detector, Phys. Lett. B 738 (2014) 428 [arXiv: 1407.8150] [INSPIRE].

[129] ATLAS collaboration, Search for an additional, heavy Higgs boson in the $H \rightarrow Z Z$ decay channel at $\sqrt{s}=8 \mathrm{TeV}$ in pp collision data with the ATLAS detector, Eur. Phys. J. C 76 (2016) 45 [arXiv: 1507.05930] [INSPIRE].

[130] M. Dhuria and G. Goswami, Perturbativity, vacuum stability and inflation in the light of $750 \mathrm{GeV}$ diphoton excess, arXiv: 1512.06782 [INSPIRE].

[131] A. Salvio and A. Mazumdar, Higgs Stability and the 750 GeV Diphoton Excess, arXiv: 1512.08184 [INSPIRE].

[132] Y. Hamada, T. Noumi, S. Sun and G. Shiu, An O(750) GeV Resonance and Inflation, arXiv:1512.08984 [INSPIRE].

[133] ATLAS collaboration, Search for high-mass diboson resonances with boson-tagged jets in proton-proton collisions at $\sqrt{s}=8 \mathrm{TeV}$ with the ATLAS detector, JHEP 12 (2015) 055 [arXiv: 1506.00962] [INSPIRE].

[134] ATLAS collaboration, Combination of searches for $W W, W Z$ and $Z Z$ resonances in $p p$ collisions at $\sqrt{s}=8 \mathrm{TeV}$ with the ATLAS detector, arXiv:1512.05099 [INSPIRE]. 
[135] B.A. Dobrescu and Z. Liu, W'Boson near 2 TeV: Predictions for Run 2 of the LHC, Phys. Rev. Lett. 115 (2015) 211802 [arXiv:1506.06736] [INSPIRE].

[136] Y. Gao, T. Ghosh, K. Sinha and J.-H. Yu, SU(2) $\times \mathrm{SU}(2) \times \mathrm{U}(1)$ interpretations of the diboson and Wh excesses, Phys. Rev. D 92 (2015) 055030 [arXiv:1506.07511] [INSPIRE].

[137] J. Brehmer, J. Hewett, J. Kopp, T. Rizzo and J. Tattersall, Symmetry Restored in Dibosons at the LHC?, JHEP 10 (2015) 182 [arXiv:1507.00013] [INSPIRE].

[138] P.S. Bhupal Dev and R.N. Mohapatra, Unified explanation of the eejj, diboson and dijet resonances at the LHC, Phys. Rev. Lett. 115 (2015) 181803 [arXiv:1508.02277] [INSPIRE].

[139] F.F. Deppisch et al., Reconciling the $2 \mathrm{TeV}$ excesses at the LHC in a linear seesaw left-right model, Phys. Rev. D 93 (2016) 013011 [arXiv: 1508.05940] [InSPIRE].

[140] F. Dias et al., Combination of Run-1 Exotic Searches in Diboson Final States at the LHC, arXiv: 1512.03371 [INSPIRE].

[141] J. Brehmer et al., The Diboson Excess: Experimental Situation and Classification of Explanations; A Les Houches Pre-Proceeding, arXiv:1512.04357 [INSPIRE].

[142] M.-L. Xiao and J.-H. Yu, Stabilizing electroweak vacuum in a vectorlike fermion model, Phys. Rev. D 90 (2014) 014007 [arXiv:1404.0681] [INSPIRE].

[143] D0 collaboration, V.M. Abazov et al., An Improved determination of the width of the top quark, Phys. Rev. D 85 (2012) 091104 [arXiv:1201.4156] [INSPIRE].

[144] CDF collaboration, T.A. Aaltonen et al., Direct Measurement of the Total Decay Width of the Top Quark, Phys. Rev. Lett. 111 (2013) 202001 [arXiv: 1308.4050] [INSPIRE]. 